\title{
TU/e EnNHOUN

\section{Highly circularly polarized broad-band emission from chiral naphthalene diimide-based supramolecular aggregates}

\section{Citation for published version (APA):}

Salerno, F., Berrocal, J. A., Haedler, A. T., Zinna, F., Meijer, E. W., \& Di Bari, L. (2017). Highly circularly polarized broad-band emission from chiral naphthalene diimide-based supramolecular aggregates. Journal of Materials Chemistry C, 5(14), 3609-3615. https://doi.org/10.1039/c7tc00281e

DOI:

$10.1039 / \mathrm{c} 7 \mathrm{tc00281e}$

Document status and date:

Published: 14/04/2017

\section{Document Version:}

Typeset version in publisher's lay-out, without final page, issue and volume numbers

\section{Please check the document version of this publication:}

- A submitted manuscript is the version of the article upon submission and before peer-review. There can be important differences between the submitted version and the official published version of record. People interested in the research are advised to contact the author for the final version of the publication, or visit the $\mathrm{DOI}$ to the publisher's website.

- The final author version and the galley proof are versions of the publication after peer review.

- The final published version features the final layout of the paper including the volume, issue and page numbers.

Link to publication

\section{General rights}

Copyright and moral rights for the publications made accessible in the public portal are retained by the authors and/or other copyright owners and it is a condition of accessing publications that users recognise and abide by the legal requirements associated with these rights.

- Users may download and print one copy of any publication from the public portal for the purpose of private study or research.

- You may not further distribute the material or use it for any profit-making activity or commercial gain

- You may freely distribute the URL identifying the publication in the public portal.

If the publication is distributed under the terms of Article 25fa of the Dutch Copyright Act, indicated by the "Taverne" license above, please follow below link for the End User Agreement:

www.tue.nl/taverne

Take down policy

If you believe that this document breaches copyright please contact us at:

openaccess@tue.nl

providing details and we will investigate your claim. 


\title{
Journal Name
}

\section{ARTICLE}

\section{Highly circularly polarized broad-band emission from chiral naphthalene diimide-based supramolecular aggregates}

Received 00th January 20xx, Accepted 00th January 20xx

DOI: $10.1039 / \times 0 \times x 00000 x$

www.rsc.org/

\author{
Francesco Salerno, ${ }^{a, b}$ José Augusto Berrocal, ${ }^{b}$ Andreas T. Haedler, ${ }^{b}$ Francesco Zinna, ${ }^{a}$ E. W. Meijer ${ }^{b}$ \\ and Lorenzo Di Bari ${ }^{\mathrm{a}}$
}

\begin{abstract}
We report off-white Circularly Polarized Luminescence (CPL) from supramolecular aggregates formed by a chiral carboxylic acid-functionalized naphthalene diimide. The self-assembly behaviour and the stability of the aggregates in solution were assessed by variable-temperature UV-Vis and Electronic Circular Dichroism experiments. The observed 200 $\mathrm{nm}$-wide emission band is unusual for a single chromophore and is a consequence of the supramolecular ordering due to a combination of $\pi$-stacking and H-bonding interactions, leading to excimer formation. High emission anisotropy factors $g_{\text {lum }}$, around $|1-2| \times 10^{-2}$, were observed over the whole spectrum in chlorinated/hydrocarbon solvent mixtures, as well as in thin film. These values are one order of magnitude higher than those of most chiral self-assembled fluorophores and other common small organic emitters.
\end{abstract}

\section{Introduction}

Circularly Polarized (CP) light is gaining importance in several modern technologies such as optical-spin communications, ${ }^{1}$ molecular photoswitches, ${ }^{2}$ optical data storage $^{3}$ and noninvasive medical diagnosis techniques. ${ }^{4}$ For example, one of the most promising applications involves the use of lightemitting devices which generate intrinsically polarized light in display technology. Currently, CP-filters are used to suppress reflection of ambient light and achieve a deep black background color. In detail, external light entering the device gets circularly polarized by the CP-filter. Upon reflection at the back electrode of the device, the circular polarization is inverted and the reflected light is absorbed by the same CPfilter before it can reach the observer. ${ }^{5}$ As a consequence, however, the intensity of the unpolarized light generated within the device is let through the filter with $50 \%$ attenuation, causing a considerable loss of efficiency. If the device emits CPlight matching the filter polarization, this problem is relieved with a considerable efficiency increase, which may theoretically attain a factor $2 .{ }^{6}$ Consequently, high CP-emission would be strongly desirable.

The extent of circularly polarized luminescence (CPL) can

\footnotetext{
Dipartimento di Chimica e Chimica Industriale Università di Pisa via Moruzzi 13, I56124 Pisa, Italy E-mail: Iorenzo.dibari@unipi.it

${ }^{b .}$ Institute for Complex Molecular Systems, Eindhoven University of Technology

P.O. Box 513, $5600 \mathrm{MB}$ Eindhoven, The Netherlands.

† Footnotes relating to the title and/or authors should appear here.

Electronic Supplementary Information (ESI) available: [details of any supplementary information available should be included here]. See DOI: $10.1039 / x 0 x \times 00000 x$
}

be conveniently estimated through the dissymmetry factor $g_{\text {lum }}$ defined as:

$$
g_{\text {lum }}=\frac{\left(\mathrm{I}_{\mathrm{L}}-\mathrm{I}_{\mathrm{R}}\right)}{\frac{1}{2}\left(\mathrm{I}_{\mathrm{L}}+\mathrm{I}_{\mathrm{R}}\right)}=\frac{\Delta \mathrm{I}}{\mathrm{I}}
$$

where $I_{L}$ and $I_{R}$ are the intensities of the left and right CPcomponents of the emission, respectively. It is important to notice that $\left|g_{\text {lum }}\right| \leq 2$, with $g_{\text {lum }}= \pm 2$ meaning a complete polarization. Some lanthanide complexes display $g$ factors in the order of 0.1 to 1 with very sharp emission bands, ${ }^{7-9}$ suitable as emitters in organic light-emitting diodes (OLEDs) due to their highly $\mathrm{CP}$ electroluminescence. ${ }^{10,11}$ On the other hand, small organic molecules, oligomers or polymers typically display $g$ factors around $10^{-4}-10^{-3} .{ }^{12-16}$ It is possible to enhance their chiroptical properties, by exploiting molecular systems that self-assemble into supramolecular architectures with long-range order. ${ }^{17-19}$ Organic chromophores have intrinsically broader emission bands than lanthanides, which is desirable for some applications such as solid-state lighting and broadband amplifiers for optical fiber communication. ${ }^{20}$

Direct white light OLEDs are highly beneficial, but achieving broad-band CP emitting devices based on a single emitter is a challenge. ${ }^{21-23} \mathrm{~A}$ possible solution consists in using a combination of fluorophores emitting in different spectral zones, typically red, green and blue $e^{24,25}$ or two complementary colors. $^{26}$ This strategy results in variable degrees of polarization (g-factor) throughout the spectrum, which is undesirable because, after a CP-filter, the light would no longer result white. To the best of our knowledge, very few white circularly polarized light emitting systems have been 
developed so far and possess typically low emission dissymmetry factors. ${ }^{27,28}$

Naphthalene diimides (NDIs) are a class of $\pi$-conjugated $n$ type semiconducting small molecules with applications in organic optoelectronics and in supramolecular chemistry. ${ }^{30}$ It has been shown recently that a simple $\mathrm{N}$-desymmetrized NDI functionalized with a carboxylic acid can emit white light thanks to supramolecular organization in bulk or in suitable solvent mixtures. ${ }^{29}$ The same authors suggested that the photophysical properties may arise from aggregation modes mainly driven by chained $\mathrm{H}$-bonding between consecutive carboxylic groups in syn-syn catemer motifs, instead of the more common acid-dimers formation. ${ }^{29}$ In this paper, we introduce an enantiopure stereocentre into an $\mathrm{N}$ desymmetrized NDI (NDI 3, scheme 1). In order to characterize the stability of the aggregate and the self-assembly behavior in solution, we used variable-temperature (VT) optical techniques such as Ultraviolet-Visible (UV-Vis), Electronic Circular Dichroism (ECD) and Photoluminescence (PL). Furthermore, we show that it is possible to obtain chiral aggregates in solution and in bulk with a high CPL dissymmetry factor over the whole emission spectrum.

\section{Results and discussion}

\section{Synthesis and self-assembly characterization by UV-Vis and ECD spectroscopy}

Starting from commercially available naphthalene tetracarboxylic dianhydride (NTDA 1, Scheme 1), NDI 3 was synthesised in two steps via a modified microwave-assisted procedure. $^{31}$ Compared to the more common statistical approach, ${ }^{32,33}$ which involves the use of both amines in a onepot protocol, this faster methodology, gave higher yields and less bis-reacted by-product. (S)-3,7-dimethyloctylamine was chosen as a chiral enantiopure building block and was synthesised according to literature procedures starting from (S)-citronellol, ${ }^{34}$ an inexpensive enantiopure terpene. The product NDI 3 obtained was purified with column chromatography and fully characterised by means of proton and carbon nuclear magnetic resonance $\left({ }^{1} \mathrm{H}-\mathrm{NMR}\right.$ and ${ }^{13} \mathrm{C}$ NMR, respectively) spectroscopy, Fourier transform infrared (FT-IR) spectroscopy and matrix assisted laser desorption ionization-time of flight mass spectrometry (MALDI-TOF-MS) (SI). The self-assembly process and the stability of the supramolecular aggregates in solution were studied with a number of optical techniques, namely VT-UV-Vis/ECD/PL. NDI $\mathbf{3}$ is soluble in chlorinated solvents (good solvents, e.g. chloroform or tetrachloroethane) and self-assembles upon addition of hydrocarbon solvents (poor solvents, e.g. cyclohexane or methylcyclohexane). In a $5 \times 10^{-5} \mathrm{M} \mathrm{CHCl}_{3}$ (or TCE) solution NDI $\mathbf{3}$ is molecularly dissolved and the UV-Vis spectrum shows an intense vibrationally resolved band with three maxima at 376, 360 and $343 \mathrm{~nm}$ (band I). The structure of band I, which is characteristic for NDI, is associated to the allowed transition with the electric dipole moment polarized along the long axis of the NDI chromophore. The higher energy

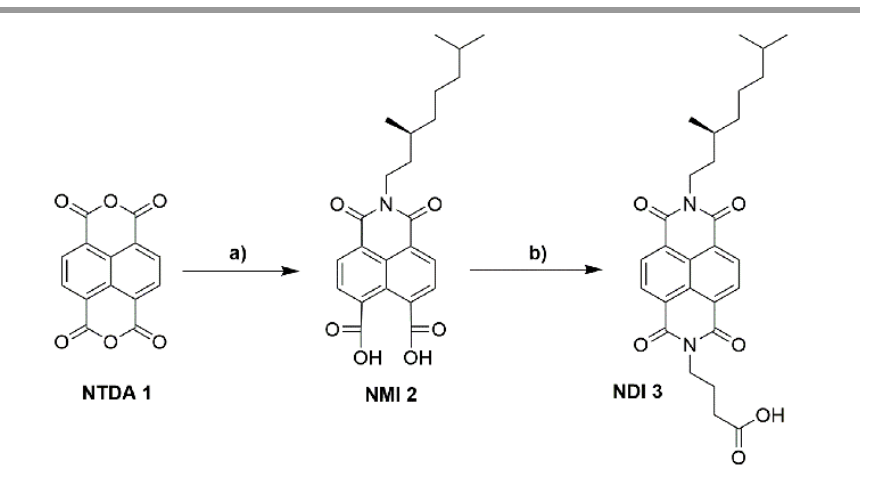

Scheme 1. Synthesis of NDI 3: a) (S)-3,7-dimethyloctylamine (1 eq), DMF, microwave (MW): $5 \mathrm{~min}$ at $75^{\circ} \mathrm{C}$, followed by $5 \mathrm{~min}$ at $140{ }^{\circ} \mathrm{C}, 47 \%$ yield; b) $\gamma$-aminobutyric acid ( 3 eq), DMF, MW: $5 \mathrm{~min}$ at $75{ }^{\circ} \mathrm{C}$ followed by $20 \mathrm{~min}$ at $140{ }^{\circ} \mathrm{C}, 43 \%$ yield.

band (band II), which is polarized along the short-axis and expected around 200-250 nm (Figure 1b), ${ }^{35,36}$ is barely visible because of the solvent cut-off. NDI 3 is insoluble in pure hydrocarbons, thus a coarse set of variable solvent compositions based on chloroform and cyclohexane (Cy) was explored to study the aggregation behavior (Figure 1a). A considerable fraction $(80 \% \mathrm{v} / \mathrm{v})$ of poor solvent was required to trigger the self-assembly process at room temperature. Consequently, a hypochromic effect on band I and the rise of a new red-shifted peak (396 nm) in the UV-Vis (Figure 1a) is observed. These spectral changes, which are consistent with previous literature reports, suggest J-type $\pi$-stacking of the chromophores. $^{37,38}$

To gain insight into the stability of the aggregates, we investigated the self-assembly at constant solvent composition, while changing the temperature. VT-UV-Vis experiments were carried out upon cooling a heated solution of NDI 3. To access higher temperatures we switched from $\mathrm{CHCl}_{3} / \mathrm{Cy}$ to higher boiling 1,1,2,2-tetrachloroethane (TCE)/metylcyclohexane (MCH). The change in solvent mixture did not significantly alter the supramolecular behavior of NDI 3 at room temperature, as confirmed with UV-Vis spectroscopy (Figure S1). The $5 \times 10^{-5} \mathrm{M}$ TCE/MCH $5 / 95$ mixture was thoroughly equilibrated at $85{ }^{\circ} \mathrm{C}$ before recording the UV-Vis spectra in $10^{\circ} \mathrm{C}$-steps upon cooling. The rise of the aggregation peak and the drop of the monomer band were observed at a critical temperature around $45^{\circ} \mathrm{C}$ (Figure 2a). The spectra also showed a quasi-isosbestic point at $385 \mathrm{~nm}$. It should be noted, that the simultaneous formation of larger supramolecular aggregates causes a scattering tail above $400 \mathrm{~nm}$. Such hardly controllable formation of macroaggregates, as combined to delicate changes in sample preparation can yield differences in spectra, which are recorded under seemingly identical conditions. $^{39,40}$

The mechanism of the self-assembly was studied by UV-Vis spectroscopy at specific wavelengths during cooling-heating cycles, at rates of $60^{\circ} \mathrm{C} / \mathrm{h}$. Starting from $80^{\circ} \mathrm{C}$ we followed the absorption intensity at $376 \mathrm{~nm}$ and $396 \mathrm{~nm}$, representing the $0-0^{\prime}$ monomer peak and the aggregation peak maxima, respectively. 


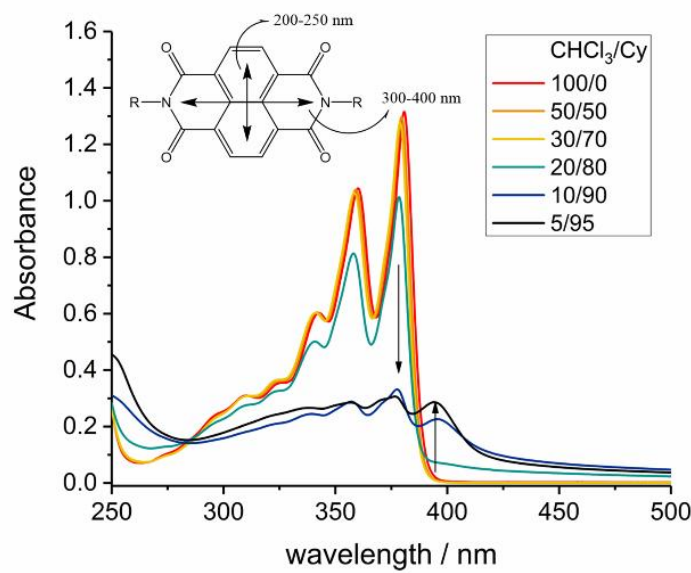

Figure 1. UV-Vis spectra of NDI 3 in different mixtures of $\mathrm{CHCl}_{3}$ and cyclohexane $(c=$ $\left.5 \times 10^{-5} \mathrm{M}, \mathrm{RT}\right)$. Spectral changes with increasing amounts of apolar solvent are indicated by arrows. Inset: scheme of the transition dipoles in the naphthalene diimide chromophore.

\section{A}

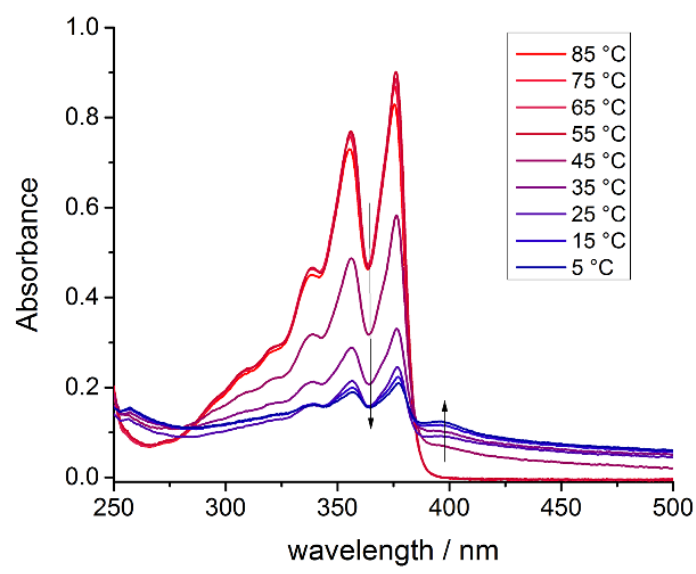

B

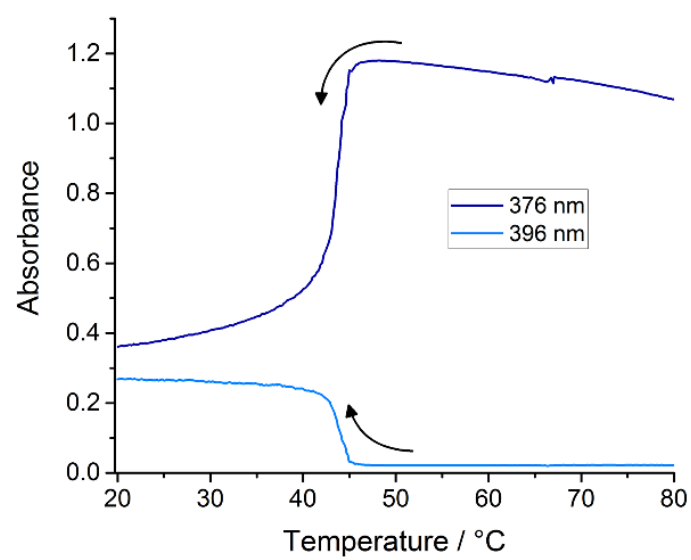

Figure 2. VT-UV-Vis spectra of NDI 3 in TCE/MCH (5/95), $\mathrm{c}=5 \times 10^{-5} \mathrm{M}$. A: upon cooling each spectrum was recorded 10 minutes after the chosen temperature was reached. B: Absorbance at 376 and $396 \mathrm{~nm}$ upon cooling from 80 to $20^{\circ} \mathrm{C}$ with a cooling rate of $60^{\circ} \mathrm{C} / \mathrm{h}$.

The cooling curves observed (Figure $2 \mathrm{~b}$ ) are non sigmoidal, which indicates a cooperative self-assembly mechanism. ${ }^{41,42}$
These results are in line with a chained $\mathrm{H}$-bonding system recently proposed for this class of molecules. ${ }^{43}$ The formation of supramolecular aggregates with defined helicity was highlighted and followed by means of VT-Electronic Circular Dichroism (ECD) spectroscopy, (Figure 3). No significant ECD signal was measured between $85^{\circ} \mathrm{C}$ and $55^{\circ} \mathrm{C}$. This situation is often encountered in similar cases, when an isolated chromophore contains a remote chiral perturber and provides a very weak chiroptical response. ${ }^{44}$ Abruptly at $45^{\circ} \mathrm{C}$ a sequence of Cotton effects became visible (Figure 3 ).

The critical aggregation temperature observed here is consistent with the VT-UV-Vis measurements previously discussed. Three regions are of interest:

I) A very broad negative feature well above $400 \mathrm{~nm}$; the shape of this feature strongly indicates a scattering effect, due to a partial precipitation of macroaggregates; interestingly, it corresponds to the tail observed in the absorption spectrum at long wavelengths in high hydrocarbon solvent content (see Figure 2a).

II) A negative Cotton effect around $400 \mathrm{~nm}$, immediately followed by a vibrationally-resolved positive band at $370 \mathrm{~nm}$, which may be interpreted as a negative exciton couplet allied to the NDI band I.

III) A strong negative band below $300 \mathrm{~nm}$, followed by the start of a positive feature unfortunately cut off by the solvent absorption, which may suggest another negative couplet connected to NDI band II.

A smaller negative ECD signal at $320 \mathrm{~nm}$ should also be mentioned. On further lowering the temperature, the Cotton effects increase only slightly in intensity while they preserve the profile shape. The ECD spectra also displayed quasiisodichroic points in proximity to the crossover wavelengths (around 345 and $395 \mathrm{~nm}$ ). The consistency of the ECD features and quasi-isosbestic and quasi-isodichroic points in the UV-Vis and ECD spectra suggest the transition of the monomers into a single type of aggregates, featuring only one expression of supramolecular chirality. Hence, we conclude that below $45^{\circ} \mathrm{C}$ a supramolecular polymer with a defined supramolecular chirality is generated in TCE/MCH 5/95 mixture. The degenerate exciton coupling and the negative sign of the couplets (regions II and III in Figure 3) suggest that both transition dipoles, i.e. short and long-axis, are arranged in $\mathrm{M}$ type helices. ${ }^{36,45}$ To study the influence of hydrogen bonding in the self-assembly of NDI $\mathbf{3}$ we added a small amount of methanol to a fully aggregated system in TCE/MCH 5/95 at room temperature. A small percentage of competing protic solvent $(0.6 \% \mathrm{v} / \mathrm{v})$ was sufficient to reduce the ECD signals to a negligible value (Figure S3), highlighting the pivotal role played by $\mathrm{H}$-bonding interactions in the self-assembly process and on the stability of the supramolecular aggregates.

Finally, additional information about the self-assembly mechanism were obtained from VT-ECD cooling-heating curves as in the VT-UV-Vis experiment discussed above (Figure $2 \mathrm{~b}$ ). The ECD signal was followed at $405 \mathrm{~nm}$, in correspondence to the minimum of the first Cotton effect. A non-sigmoidal cooling curve was observed, supporting the cooperative selfassembly mechanism (Figure S4b). ${ }^{46}$ 


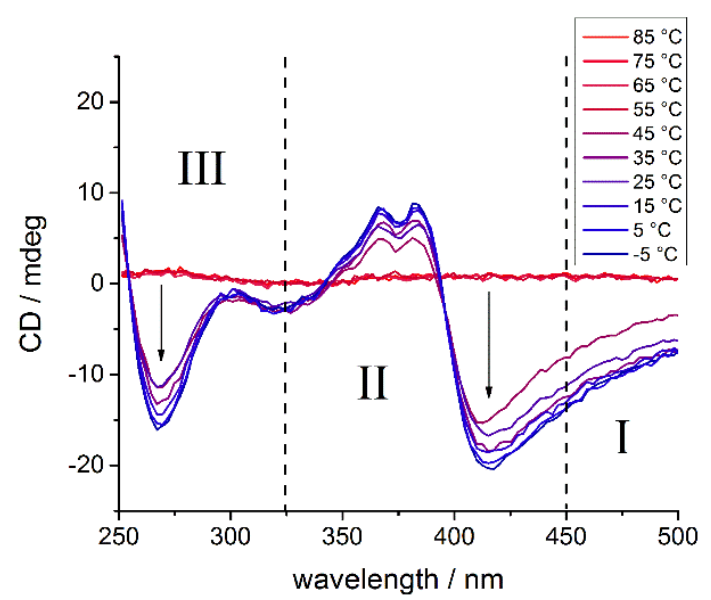

Figure 3. ECD spectra of compound NDI 3 in TCE/MCH (5/95), $c=5 \times 10^{-5} \mathrm{M}$. Upon cooling each spectrum was recorded 10 minutes after the chosen temperature had been reached.

\section{Photoluminescence and Circularly Polarized Luminescence characterization}

To compare the different photophysical properties of isolated molecules vs. aggregates, we carried out VTphotoluminescence studies on $5 \times 10^{-5} \mathrm{M} 5 / 95 \mathrm{TCE} / \mathrm{MCH}$ solution of NDI 3. The spectra were recorded upon excitation at $356 \mathrm{~nm}$ (the $0-1^{\prime}$ peak of the band I vibronic progression). Above $45^{\circ} \mathrm{C}$, where NDI 3 is in a molecularly dissolved state, the emission spectrum is structured and resembles the mirrorimage of the absorption spectrum in chloroform. In contrast, below $45{ }^{\circ} \mathrm{C}$ the monomer emission becomes less intense and a broader band in the range $450-650 \mathrm{~nm}$ arises (Figure 4 a). The broad unstructured emission band and the large Stokes shift $(\approx 75 \mathrm{~nm}$ ) suggest the formation of excimer-like species induced by ground-state aggregation. ${ }^{47,48}$ The emission profile is comparable to those reported by Ghosh et al for achiral analogous systems. ${ }^{43}$ To quantitatively evaluate the emission color of the aggregate in solution we made use of the standard CIE color coordinates (Commission Internationale de l'Éclairage). The values found $(0.26,0.34)$ for the sample at $25^{\circ} \mathrm{C}$ (Figure $4 \mathrm{~b}$ ), are extremely close to theoretical, perfectly white coordinates $(0.33,0.33)$.

Circularly Polarized Luminescence (CPL) spectra were recorded in aggregation conditions $\left(\mathrm{CHCl}_{3} / \mathrm{Cy} 5 / 95\right)$ at room temperature, with a $365 \mathrm{~nm}$ UV lamp as excitation source. ${ }^{49}$ CPL signal and total emission detected for the aggregation band are shown in Figure 5. Two negative bands can be observed in correspondence to the maxima of the excimer-like emission. The glum dissymmetry factor gives quantitative information about the circular polarization of the emitted light, resulting in around $-2 \times 10^{-2}$ for the band at $470 \mathrm{~nm}$ and $1 \times 10^{-2}$ at $555 \mathrm{~nm}$ (Figure S5a).

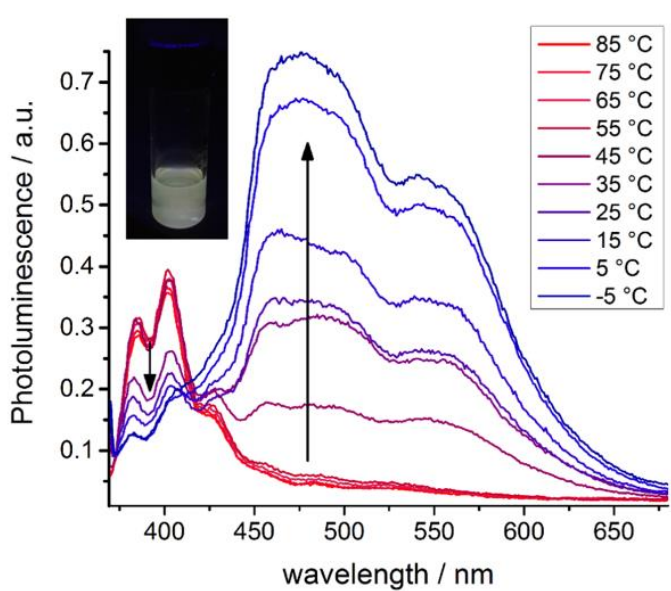

Figure 4. Photoluminescence spectra of compound NDI 3 at different temperatures in TCE $/ \mathrm{MCH}(5 / 95), c=5.0 \times 10^{-5} \mathrm{M}, \lambda_{\text {ex }}=356 \mathrm{~nm}$. Upon cooling each spectrum was recorded 10 minutes after the chosen temperature had been reached. Inset: picture of the sample at $\mathrm{RT}, \mathrm{CHCl}_{3} / \mathrm{Cyclohexane}$ (5/95), $c=5 \times 10^{-5} \mathrm{M}$, under UV (365 nm) irradiation.

These values are higher than those reported for most organic emitters, especially over such a wide wavelength range ${ }^{27,28,50}$ but are reasonable for CPL stemming from excimer bands. ${ }^{51-54}$ The large Stokes shift and the consequent separation between the absorption and emission bands ensure that there is no contribution from Circular Dichroism to the observed CPL. ${ }^{55}$

It is worth to stress that these intriguing CPL results are a consequence of the supramolecular architecture formed by a simple chiral molecule, which was easily synthesised.

Since applications in emitting devices commonly require a solid state active layer, we tested whether the emissive properties of this supramolecular structure can be obtained in thin films, as well. A concentrated $\left(10^{-3} \mathrm{M}\right)$ chloroform solution of NDI 3 was drop-casted on a quartz slide and analysed by ECD and CPL spectroscopies after solvent evaporation. Linear dichroism and inhomogeneity effects of the thin film were ruled out by recording spectra at different orientation of the sample (see SI). Two negative exciton couplets, comparable to the spectra of the aggregate in solution, were observed in the ECD spectra (Figure S6), suggesting that the same helical structure is formed even during fast evaporation of chloroform. The thin film obtained showed broad band emission as well, as shown in Figure 6a. However, the intensity ratio between the lower energy band (around $555 \mathrm{~nm}$ ) and the higher energy band (at $470 \mathrm{~nm}$ ) is reduced compared to the solution-phase spectrum. Moreover, the width of the whole emission band decreased and emission color coordinates shift to sky blue $(0.17,0.29)$. Other preparations of thin films (dispersion in a polymer matrix, annealing, etc.) shall be considered in the future in order to outweigh this effect and achieve color emission closer to real white. 


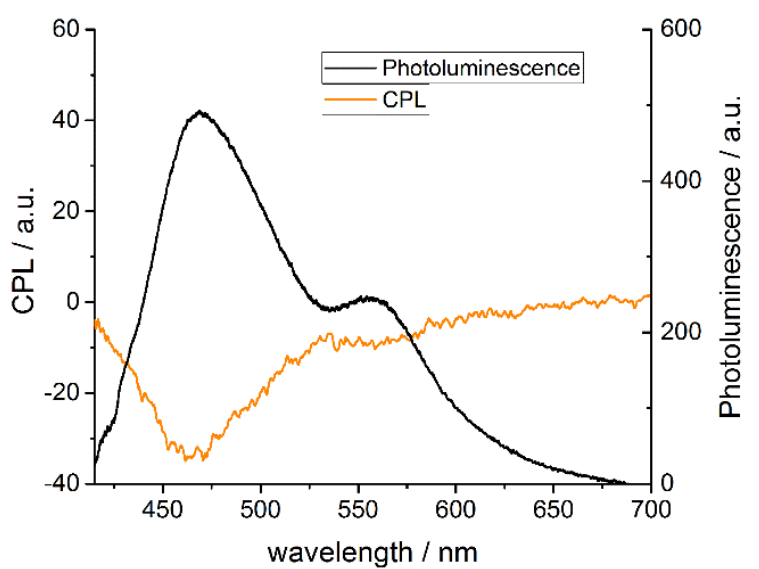

Figure 5. Photoluminescence (black) and CPL (yellow) spectra of compound NDI 3 at RT

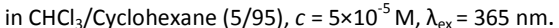
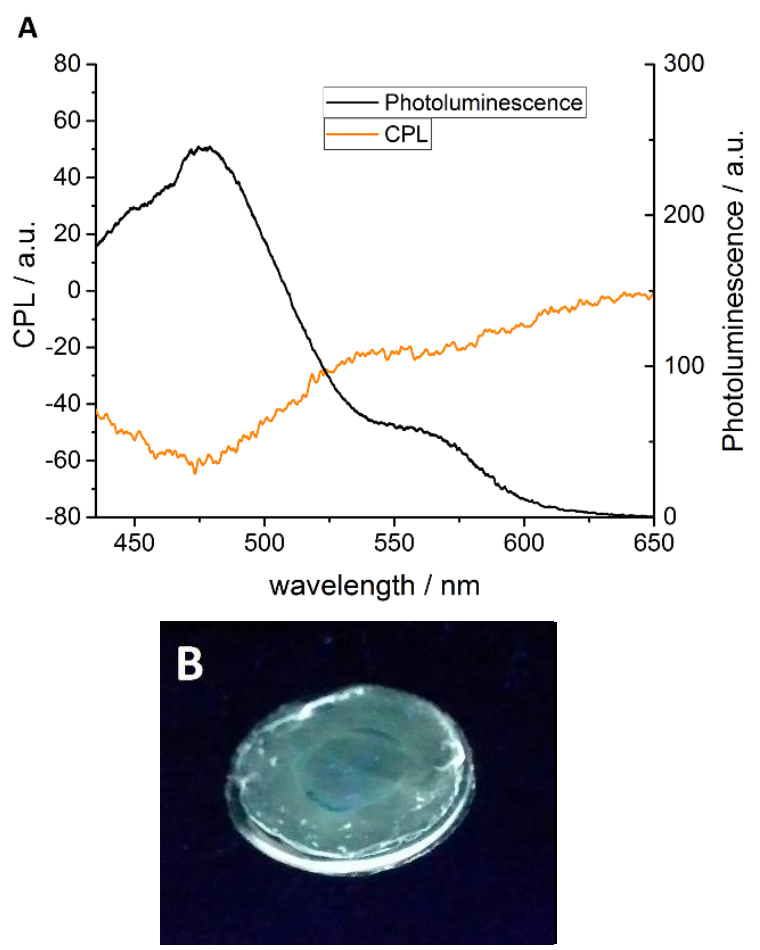

Figure 6. Photoluminescence/CPL spectra (A) and picture (B) of compound NDI 3 as thin film obtained by deposition of $\mathrm{CHCl}_{3}$ solution $10^{-3} \mathrm{M}$ under $\mathrm{UV}(365 \mathrm{~nm})$ irradiation. The emission spectra shown are averaged on three samples.

Despite these issues, remarkably high values of $\mathrm{g}_{\text {lum }}$ factor around $-1.5 \times 10^{-2}$ for the high energy band and $-2 \times 10^{-2}$ for thelow energy one, were measured (Figure S5b). The transition from solution-phase to solid state did not heavily affect the chiral order of the supramolecular system. Even though solid-state emission resulted somewhat shifted, the chiral aggregates of NDI 3 retained the unusual property of a broad band and noticeably high emission dissymmetry factor.

\section{Morphological study of drop-casted thin films of NDI 3}

To uncover the possible differences between the aggregates obtained in solution and in the solid state, which give rise to the Photoluminescence and CPL spectra shown in Figures 5 and $6 a$, we investigated the morphologies of the drop-casted films from $5 / 95 \mathrm{TCE} / \mathrm{MCH}$ and $\mathrm{CHCl}_{3}$ with Scanning Electron Microscopy (SEM) and Transmission Electron Microscopy (TEM). Stock solutions of NDI 3 in the two solvents were prepared at a $10^{-3} \mathrm{M}$ nominal concentration: while in $5 / 95 \mathrm{TCE} / \mathrm{MCH}$ the majority of the material did not dissolve, in $\mathrm{CHCl}_{3}$ we obtained a clear solution. The TCE/MCH suspension was sonicated and the undissolved material was left to sediment at the bottom of the vial. White luminescence was observed from the supernatant of the $5 / 95 \mathrm{TCE} / \mathrm{MCH}$ mixture after excitation with $365 \mathrm{~nm}$ UV light, while no emission was detected from the $\mathrm{CHCl}_{3}$ solution.

Characteristic SEM micrographs measured for the aggregates of NDI 3 in 5/95 TCE/MCH and $\mathrm{CHCl}_{3}$ are shown in Figure $7 \mathrm{a}$ and $7 \mathrm{~b}$, respectively. Additional SEM micrographs for both solvents are available in ESI. Regardless of the solvent used, a compact and homogeneous layer is created at the interface with the substrate (glass), above which the excess of material appears to form corrugated and polymorph crystalline domains separated by grain boundaries (Figure 7a and $7 \mathrm{~b}$ ). The crystallization above the uniform layer seems more pronounced in the $\mathrm{CHCl}_{3}$ sample (Figure 7b) than in the $5 / 95 \mathrm{TCE} / \mathrm{MCH}$ one (Figure 7a), most likely due to a higher concentration of NDI 3 in the $\mathrm{CHCl}_{3}$ solution. Such difference between the two samples is also visible by eye, with the film obtained from 5/95 TCE/MCH being more flat and uniform and that from $\mathrm{CHCl}_{3}$ being more corrugated and thick. The white luminescence was observed for both films upon $365 \mathrm{~nm}$ excitation, which contrast starkly with the two stock solutions (vide supra). We conclude that while the $5 / 95 \mathrm{TCE} / \mathrm{MCH}$ film is partially pre-assembled in solution, the one from $\mathrm{CHCl}_{3}$ gets only generated upon slow evaporation of the solvent. As a final note, the lower regularity of the drop-casted films from $\mathrm{CHCl}_{3}$ does not seem to play a role in the emission properties of NDI 3, since the white emission upon $365 \mathrm{~nm}$ excitation originates from both the uniform layer underneath and the small crystalline protuberances. A closer inspection to the the drop-casted films by means of TEM revealed more details about their morphology (Figure 7c and 7d; ESI). Under both experimental conditions NDI $\mathbf{3}$ forms few micrometer-sized aggregates, resembling plates. Such observation seems to support the syn-syn catemer motif proposed by Ghosh ${ }^{29}$ for the self-assembly of this particular type of NDI derivatives. The plates appear larger and characterized by a dominant growth direction in 5/95 TCE/MCH (Figure 7c), while they are less extended, yet more regular in $\mathrm{CHCl}_{3}$ (Figure 7d). Consistently with the SEM measurements, the higher concentration of NDI 3 in $\mathrm{CHCl}_{3}$ allows for the formation of more extended networks of plates, separated by grain boundaries. We hypothesize that the differences in size and shape of the aggregates formed in the two experimental conditions are responsible for the minor 


\section{Journal Name}

A

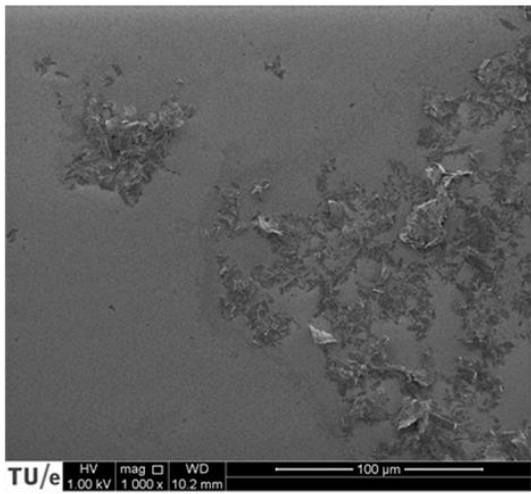

C

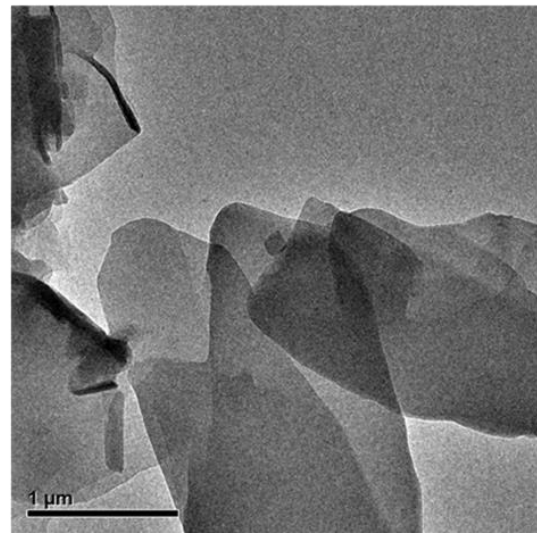

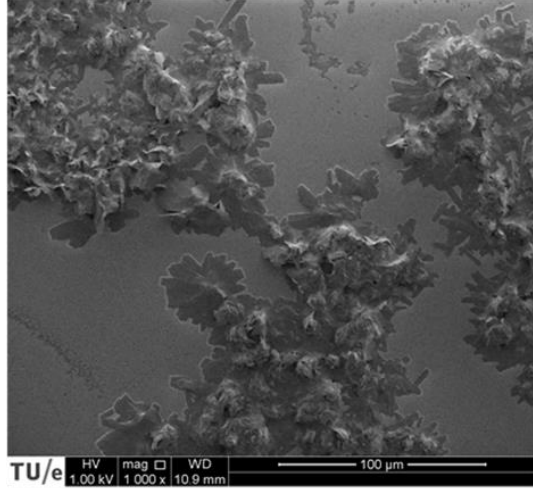

D

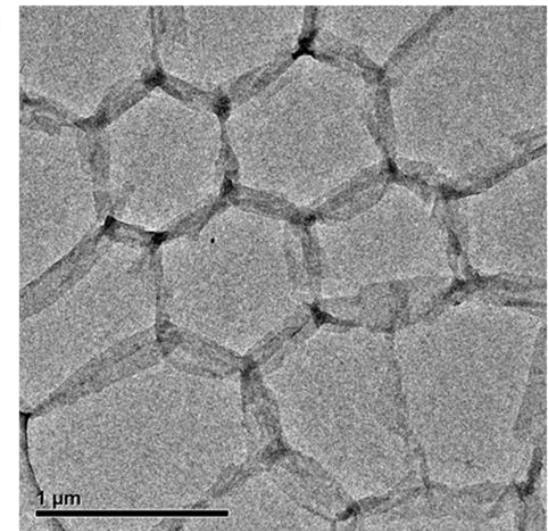

Figure 7. Morphological study of drop-casted films of NDI 3. SEM micrographs of NDI 3 in 5/95 TCE/MCH (A) and CHCl 3 (B), drop cast on glass cover slip (scale bar $100 \mu$ m). TEM micrographs of NDI 3 in 5/95 TCE/MCH (C) and $\mathrm{CHCl} 3$ (D), drop cast on TEM grids (scale bar $1 \mu \mathrm{m}$ ).

emission color change, the different ECD, and to a lesser extent CPL responses of NDI 3 in solution and film state.

\section{Conclusions}

In conclusion, we reported the synthesis and self-assembly behavior of a naphthalene diimide functionalized with a chiral pendant. The resulting supramolecular polymers are characterized by remarkable emission dissymmetry factors, which lie in the $\left|1-2 \times 10^{-2}\right|$ range over a broad spectral width (from 400 to $600 \mathrm{~nm}$ ) in both hydrocarbon-rich solvents and solid state. Such reported emission dissymmetry factors are significantly higher than those of small organic molecules reported so far. These intriguing results are rationalized by the formation of chiral supramolecular aggregates due to a synergy of $\pi$-stacking and directional $\mathrm{H}$-bonds via a cooperative self-assembly mechanism. The intermolecular interactions direct the supramolecular process leading to uncommon photophysical and chiroptical properties.

The morphological study of the thin films drop-casted from two different solvents highlighted differences in size and shape of the supramolecular aggregates formed at the nano-/micrometer length scale.

The performance of the presented desymmetrized naphthalene diimide will be further tested in a simple solid state CP-emitting device. Our investigation confirms that the introduction of chirality into functional supramolecular systems yields intriguing photophysical behaviors and can be considered a promising and convenient approach towards efficient CPL emitting systems.

\section{Acknowledgements}

We acknowledge Bas van Genabeek for synthesising and generously providing us the (S)-3,7-dimethyloctylamine. We 
also wish to thank Beatrice Adelizzi for support and discussions. The authors are thankful to Miss Matilde Putti and Miss Anneloes Oude Vrielink for SEM and TEM imaging, respectively. JAB, ATH, and EWM would like to thank the Dutch Ministry of Education, Culture and Science (Gravity program 024.001.035) and The Netherlands Organisation for Scientific Research (NWO TOP-PUNT 718.014.003) for financial support. FS thanks the Erasmus Mobility Program and, together with FZ and LDB, the University of Pisa (PRA 2016_50).

\section{Notes and references}

1 R. Farshchi, M. Ramsteiner, J. Herfort, A. Tahraoui and H. T. Grahn, Appl. Phys. Lett., 2011, 98.

2 H. Hayasaka, T. Miyashita, K. Tamura and K. Akagi, Adv. Funct. Mater., 2010, 20, 1243-1250. C. D. Stanciu, F. Hansteen, A. V. Kimel, A. Kirilyuk, A Tsukamoto, A. Itoh and T. Rasing, Phys. Rev. Lett., 2007, 99, 1-4. B. Kunnen, C. Macdonald, A. Doronin, S. Jacques, M. Eccles and I. Meglinski, J. Biophotonics, 2015, 8, 317-23. B. C. Kim, Y. J. Lim, J. H. Song, J. H. Lee, K.-U. Jeong, J. H. Lee, G.-D. Lee and S. H. Lee, Opt. Express, 2014, 22, A1725$-A 1730$. J. R. Brandt, X. Wang, Y. Yang, A. J. Campbell and M. J. Fuchter, J. Am. Chem. Soc., 2016, 138, 9743-9746. J. Kido and Y. Okamoto, Chem. Rev., 2002, 102, 2357-2368. S. V Eliseeva and J.-C. G. Bünzli, Chem. Soc. Rev., 2010, 39, 189-227.

F. Zinna and L. Di Bari, Chirality, 2015, 27, 1-13.

0 F. Zinna, U. Giovanella and L. Di Bari, Adv. Mater., 2015, 27, 1791-1795.

1 F. Zinna, M. Pasini, F. Galeotti, C. Botta, L. Di Bari and U. Giovanella, Adv. Funct. Mater., 2016.

12 B. M. W. Langeveld-Voss, D. Beljonne, Z. Shuai, R. a J. Janssen, S. C. J. Meskers, E. W. Meijer and J.-L. Brédas, Adv. Mater., 1998, 10, 1343-1348.

3 K. Suda and K. Akagi, Macromolecules, 2011, 44, 94739488.

E. M. Sánchez-Carnerero, A. R. Agarrabeitia, F. Moreno, B. L. Maroto, G. Muller, M. J. Ortiz and S. De La Moya, Chem. A Eur. J., 2015, 21, 13488-13500.

15 T. Aida, E. W. Meijer and S. I. Stupp, Science (80-. )., 2012, 335, 813-817.

16 E. Peeters, M. P. T. Christiaans, R. A. J. Janssen, H. F. M. Schoo, H. P. J. M. Dekkers and E. W. Meijer, J. Am. Chem. Soc., 1997, 119, 9909-9910.

17 T. Kaseyama, S. Furumi, X. Zhang, K. Tanaka and M. Takeuchi, Angew. Chemie - Int. Ed., 2011, 50, 3684-3687.

18 Anuradha, D. D. La, M. Al Kobaisi and S. V. Bhosale, Sci. Rep., 2015, 5, 15652.

19 S. V. Bhosale, . A., D. D. La, M. Al Kobaisi and A. Gupta, Chem. - A Eur. J., 2017, Accepted Author Manuscript. doi:10.1002/chem.20160. G. Qian and Z. Y. Wang, Chem. - An Asian J., 2010, 5, 10061029.
G. Li, T. Fleetham and J. Li, Adv. Mater., 2014, 26, 29312936.

J. Jiang, Y. Xu, W. Yang, R. Guan, Z. Liu, H. Zhen and Y. Cao, Adv. Mater., 2006, 18, 1769-1773.

Y. Liu, M. Nishiura, Y. Wang and Z. Hou, J. Am. Chem. Soc., 2006, 128, 5592-5593.

Y. Xiong, W. Xu, C. Li, B. Liang, L. Zhao, J. Peng, Y. Cao and J. Wang, Org. Electron. physics, Mater. Appl., 2008, 9, 533538.

H. Zhang, X. Shan, L. Zhou, P. Lin, R. Li, E. Ma, X. Guo and S. Du, J. Mater. Chem. C, 2013, 1, 888-891.

C. H. Chang, C. C. Chen, C. C. Wu, S. Y. Chang, J. Y. Hung and Y. Chi, Org. Electron. physics, Mater. Appl., 2010, 11, 266-272.

K. Watanabe, T. Sakamoto, M. Taguchi, M. Fujiki and T. Nakano, Chem. Commun., 2011, 47, 10996. K. Watanabe, I. Osaka, S. Yorozuya and K. Akagi, Chem. Mater., 2012, 24, 1011-1024.

M. R. Molla and S. Ghosh, Chem. - A Eur. J., 2012, 18, 1290-1294.

M. Al Kobaisi, S. V. Bhosale, K. Latham, A. M. Raynor and S. V. Bhosale, Chem. Rev., 2016, 116, 11685-11796.

K. Tambara, N. Ponnuswamy, G. Hennrich and G. D. Pantoş, J. Org. Chem., 2011, 76, 3338-3347.

Y. Hu, Y. Qin, X. Gao, F. Zhang, C. A. Di, Z. Zhao, H. Li and D. Zhu, Org. Lett., 2012, 14, 292-295.

M. R. Molla and S. Ghosh, Chem. - A Eur. J., 2012, 18, 9860-9869.

T. Terashima, T. Mes, T. F. A. De Greef, M. A. J. Gillissen, P. Besenius, A. R. A. Palmans and E. W. Meijer, J. Am. Chem. Soc., 2011, 133, 4742-5.

H. Yamaguchi, K. Kitano and K. Toyoda, Spectrochim Acta, 1982, 38, 261-263.

H. Shao, T. Nguyen, N. C. Romano, D. A. Modarelli and J. R. Parquette, J. Am. Chem. Soc., 2009, 131, 16374-16376. H. Shao, J. Seifert, N. C. Romano, M. Gao, J. J. Helmus, C. P. Jaroniec, D. A. Modarelli and J. R. Parquette, Angew. Chemie - Int. Ed., 2010, 49, 7688-7691.

F. Würthner, T. E. Kaiser and C. R. Saha-Möller, Angew. Chemie - Int. Ed., 2011, 50, 3376-3410.

A. T. Haedler, S. C. J. Meskers, R. H. Zha, M. Kivala, H. W. Schmidt and E. W. Meijer, J. Am. Chem. Soc., 2016, 138, 10539-10545.

S. Ogi, K. Sugiyasu, S. Manna, S. Samitsu and M. Takeuchi, Nat. Chem., 2014, 6, 188-95.

P. A. Korevaar, S. J. George, A. J. Markvoort, M. M. J. Smulders, P. a. J. Hilbers, A. P. H. J. Schenning, T. F. a. De Greef and E. W. Meijer, Nature, 2012, 481, 492-496. M. M. J. Smulders, M. M. L. Nieuwenhuizen, T. F. A. De Greef, P. Van Der Schoot, A. P. H. J. Schenning and E. W. Meijer, Chem. - A Eur. J., 2010, 16, 362-367.

M. R. Molla, D. Gehrig, L. Roy, V. Kamm, A. Paul, F. Laquai and S. Ghosh, Chem. - A Eur. J., 2014, 20, 760-771. G. Pescitelli, L. Di Bari and N. Berova, Chem. Soc. Rev., 2014, 43, 5211-33.

J. Gawroński, M. Brzostowska, K. Kacprzak, H. Kołbon and P. Skowronek, Chirality, 2000, 12, 263-268. 
M. M. J. Smulders, A. P. H. J. Schenning and E. W. Meijer, J. Am. Chem. Soc., 2008, 130, 606-611.

47 M. Kumar and S. J. George, Nanoscale, 2011, 3, 2130-3.

48 A. T. Haedler, H. Misslitz, C. Buehlmeyer, R. Q. Albuquerque, A. Köhler and H. W. Schmidt, ChemPhysChem, 2013, 14, 1818-1829.

49 F. Zinna, T. Bruhn, C. A. Guido, J. Ahrens, M. Bröring, L. Di Bari and G. Pescitelli, Chem. - A Eur. J., 2016, 22, 1608916098.

50 T. Ikeda, T. Masuda, T. Hirao, J. Yuasa, H. Tsumatori, T. Kawai and T. Haino, Chem. Commun., 2012, 48, 6025.

51 H. Akita, S. P. Tanis, M. Adams, V. Balogh-Nair and K. Nakanishi, JACS, 1980, 102, 6372-6374.

52 M. Inouye, K. Hayashi, Y. Yonenaga, T. Itou, K. Fujimoto, T. Uchida, M. Iwamura and N. Koichi, Angew. Chemie - Int. Ed., 2014, 53, 14392-14396.

53 K. Kano, H. Matsumoto, S. Hashimoto, M. Sisido and Y. Imanishi, J. Am. Chem. Soc., 1985, 107, 6117-6118.

54 J. Kumar, T. Nakashima, H. Tsumatori, M. Mori, M. Naito and T. Kawai, Chem. - A Eur. J., 2013, 19, 14090-14097.

55 E. Castiglioni, S. Abbate, F. Lebon and G. Longhi, Chirality, 2012, 24, 725-30. 